\title{
Assessment of Quadratic Nonlinear Cardiorespiratory Couplings During Tilt Table Test by Means of Real Wavelet Biphase
}

\author{
Spyridon Kontaxis, Jesús Lázaro, Eduardo Gil, Pablo Laguna, Raquel Bailón
}

\begin{abstract}
Objective: In this paper a method for assessment of Quadratic Phase Coupling (QPC) between respiration and Heart Rate Variability (HRV) is presented. Methods: First, a method for QPC detection is proposed named Real Wavelet Biphase (RWB). Then, a method for QPC quantification is proposed based on the Normalized Wavelet Biamplitude (NWB). A simulation study has been conducted to test the reliability of RWB to identify QPC, even in the presence of constant delays between interacting oscillations, and to discriminate it from Quadratic Phase Uncoupling. Significant QPC was assessed based on surrogate data analysis. Then, quadratic cardiorespiratory couplings were studied during a tilt table test protocol of 17 young healthy subjects. Results: Simulation study showed that RWB is able to detect even weak QPC with delays in the range of $0-2 \mathbf{s}$, which are usual in the Autonomic Nervous System (ANS) control of heart rate. Results from the database revealed a significant reduction $(p<0.05)$ of NWB between respiration and both low and high frequencies of HRV in head-up tilt position compared to early supine. Conclusion: The proposed technique detects and quantifies robustly QPC and is able to track the coupling between respiration and various HRV components during ANS changes. Significance: The proposed method can help to assess alternations of nonlinear cardiorespiratory interactions related to ANS dysfunction and physiological regulation of HRV in cardiovascular diseases.
\end{abstract}

Index Terms-Cardiorespiratory coupling, heart rate variability, respiration, wavelet bicoherence, wavelet biphase

\section{INTRODUCTION}

$\mathbf{T}$ HE coupling of heart rate (HR) to the respiratory cycle has been extensively studied as the heart period decreases during inspiration and increases during expiration [1]. These

This work was funded under projects TIN2014-53567-R by MINECO (Spain) and by BSICOS Group (T96) from Government of Aragón and European Social Fund (EU). This work was supported by CIBER which is a center of the Instituto de Salud Carlos III in assistance from the European Regional Development Fund. This project has received funding from the European Union's Framework Programme for Research and Innovation Horizon 2020 (2014-2020) under the Marie Skodowska-Curie Grant Agreement No. 745755. The computation was performed by the ICTS NANBIOSIS, more specifically by the High Performance Computing Unit of the CIBER in Bioengineering, Biomaterials \& Nanomedicine (CIBER-BBN) at the University of Zaragoza. Asterisk indicates corresponding author.

* S. Kontaxis, J. Lázaro, E. Gil, P. Laguna, R. Bailón are with the Biomedical Signal Interpretation and Computational Simulation (BSICoS) group at the Aragón Institute of Engineering Research (I3A), IIS Aragón, University of Zaragoza, Spain, (e-mail: sikontax@unizar.es, rbailon@unizar.es)

E. Gil, P. Laguna, R. Bailón are CIBER de Bioingeniería, Biomateriales y Nanomedicina (CIBER-BBN), Spain.

J. Lázaro is with Department of Biomedical Engineering, University of Connecticut, Storrs, CT 06269, USA.

Copyright (c) 2017 IEEE. Personal use of this material is permitted. However, permission to use this material for any other purposes must be obtained from the IEEE by sending an email to pubs-permissions@ieee.org. rhythmical fluctuations in heart periods are known as respiratory sinus arrhythmia (RSA) [2]. The RSA is mediated by the parasympathetic nervous system via the vagus nerve [3] and has been used as an index, although partially incomplete [4], of cardiac vagal tone [5]. Heart rate variability (HRV) has been proposed as a noninvasive indicator of autonomic nervous system (ANS) regulation over the heart [6] and RSA is usually reflected in high-frequency oscillations in the typical frequency range $(0.15-0.4 \mathrm{~Hz})$ of normal adult respiration.

HRV is often considered to have a complex structure involving various superimposed oscillations, nonlinearly related to each other [7]. RSA may be influenced by respiratory pacemaker oscillations in central nervous system, which occasionally differ from actual breathing. The pacemaker hypothesis posits that a kernel of neurons with nonlinear intrinsic bursting properties are essential to generate synchronized rhythmic activity, that is distributed to follower respiratory neurons [8], [9]. Both pacemaker and actual respiration may produce heart rate oscillations [10]. The central modulation of sympathetic nerve activity by respiration, occurs due to coupling of presympathetic centers in the medulla oblongata with central pattern generators of respiration [11], [12].

Anatomical studies have demonstrated that the generators of cardiac and respiratory rhythm are located in the brainstem and thus the interaction between cardiovascular and respiratory systems is affected by the activity of higher brain regions of central nervous system [13], [14]. In [15] causal relationships between cardiac, respiratory and brain oscillatory processes were reported in rats, varying with the state of anesthesia. Furthermore, it has been demonstrated that there is no synchronization between both systems in coma or heavily sedated subjects ventilated via a respirator. In these situations, the cardiac and respiratory systems seemed to be disconnected, resulting in cardiac oscillations of constant frequency and no relationship between their phases [16]. A variety of studies suggest that the cardiorespiratory system exhibits nonlinear interactions between breathing and heart rate [17]-[19]. Nonlinear nature between external stimuli and heart period fluctuations can be attributed to the nonlinear features of the sinoatrial (SA) node [20], among others.

Cardiorespiratory nonlinear interactions have been addressed by analyses including nonlinear prediction [21], entropy [22], symbolization [23], phase synchronization [24] and recurrence quantification analysis [25]. Information theory has proven to be useful to evaluate directional interactions in cardiorespiratory data [26] and it has been found that those 
interactions are mostly unidirectional from respiration to HRV [27]. The tendency of unidirectional coupling from respiratory to cardiovascular system has been found also in terms of mutual predictability considering weak coupling between two self-sustained oscillators from bivariate data [28], [29].

In [30] the respiratory and cardiac frequencies and phases were used for evaluating the direct influence of respiration on the heart. Assessing the cardiorespiratory coupling function and its time evolution, the mechanism through which respiration influences the cardiac oscillations can be revealed. Nonlinear properties of mechanisms which generate time series via phase relations of their harmonic components can be quantified using High Order Statistics (HOS) analysis [31]. Phase information of characteristic frequencies is able to characterize the nonlinear nature, e.g. quadratic or cubic, of interacting oscillations. A variety of studies in HOS field have been implemented to analyze the nonlinear properties of HRV signals [32], [33]. In [17] and [34] a bispectral analysis has been applied to cardiorespiratory data in order to analyze the coupling mechanisms between respiratory and cardiac systems. These techniques measure time-phase relationships among interacting oscillations with quadratic nature.

Wavelets introduction in bispectrum field reduced time averages to a minimum, allowing the application of HOS in non-stationary biomedical signals [35]. A drawback of wavelet bispectrum is that its value depends on both the degree of phase coupling and the amplitude of interacting frequency components. In the case of normalized wavelet bispectrum, known as wavelet bicoherence (WB), the amplitude dependence is removed [36]. It has been demonstrated that WB does not reliably measure quadratic phase coupling (QPC), since high bicoherence levels are present also when QPC is absent [37]. Biphase Randomization Wavelet Bicoherence (BRWB), a modification of WB proposed by Li et al. [38], dealt with the problem of previous method. In the present study a novel method named Real Wavelet Biphase (RWB), which takes into account only phase information, is alternatively proposed, overcoming the previous limitations, as well as simplifying the computational complexity.

The aim of this study consists in QPC assessment between respiration and HRV during a tilt table test in a robust and reliable way. Reliability of RWB in QPC detection will be proven in a simulation study even when constant delays between components that might appear in real signals, are present. Significant QPC will be assessed based on a surrogate data analysis. Then, method's ability to discriminate different strengths of QPC from linear and, what we will later call Quadratic Phase Uncoupling, will be tested. Robustness of strength quantification will be proven for the Normalized Wavelet Biamplitude (NWB), where the amplitude dependence on the interacting oscillations has been mitigated.

The rest of the paper is organized as follows. In Section II the definition of existing methods for QPC assessment and their limitations are firstly described. Then, the proposed methods for QPC detection and quantification are presented. Subsequently, a strategy to study cardiorespiratory interactions during ANS changes induced by tilt table test, as well as and the materials used in this study are presented. A simulation study is designed to evaluate the performance of algorithms. Results are presented and discussed in Section III and Section IV, respectively. Finally, conclusions of this work are given in Section V.

\section{Methods FOR QPC DETECTION}

QPC arises when oscillations interact through a QuadraticLinear (Q-L) system (Fig. 1). A simple nonlinearity introduces new harmonics with higher-order frequency and phase correlations which simply could not have been produced through a linear transformation. This specific class of second-order nonlinear interaction involves frequency triplets $f_{1}, f_{2}, f_{1}+f_{2}$, where the sum of the phases at $f_{1}\left(\phi_{1}\right)$ and $f_{2}\left(\phi_{2}\right)$ is the phase at frequency $f_{1}+f_{2}$ (i.e. $\phi_{1}+\phi_{2}$ ) [39]. This QPC is to be distinguished from other two cases, the linear relation where no component appears at $f_{1}+f_{2}$, and what we call Quadratic Phase Uncoupling (QPU), where there are components at the triplet $f_{1}, f_{2}$ and $f_{1}+f_{2}$ but the QPC relationship is not met. The subsequent methods deal with the detection of QPC.

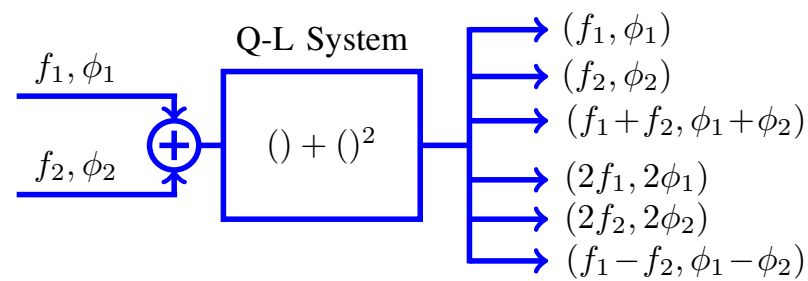

Fig. 1. The block diagram of a Quadratic-Linear (Q-L) system and the harmonics generated by 2 interacting oscillations $\left(f_{1}, f_{2}\right)$.

\section{A. Wavelet Bicoherence and Biphase Randomization Wavelet Bicoherence}

Bispectral analysis, which is used to analyze just one signal, can be extended to study nonlinear relationships between different signals $x(t), y(t), z(t)$ introducing their Continuous Wavelet Transform (CWT) coefficients $W_{x}(f, \tau), W_{y}(f, \tau)$ and $W_{z}(f, \tau)$, respectively. The wavelet cross-bispectrum $B\left(f_{1}, f_{2}\right)$ (WBS) consists of wavelet biamplitude $\left(A_{\mathrm{B}}\left(f_{1}, f_{2}\right)\right)$ and wavelet biphase $\left(\Phi_{\mathrm{B}}\left(f_{1}, f_{2}\right)\right)$ which are given by [35]:

$$
\begin{aligned}
B\left(f_{1}, f_{2}\right) & =\int_{T} W_{x}\left(f_{1}, \tau\right) W_{y}\left(f_{2}, \tau\right) W_{z}^{*}\left(f_{12}, \tau\right) \mathrm{d} \tau \\
& =\int_{T} A_{\mathrm{w}}\left(f_{1}, f_{2}, \tau\right) e^{j \Phi_{\mathrm{W}}\left(f_{1}, f_{2}, \tau\right)} \mathrm{d} \tau \\
& =A_{\mathrm{B}}\left(f_{1}, f_{2}\right) e^{j \Phi_{\mathrm{B}}\left(f_{1}, f_{2}\right)}
\end{aligned}
$$

where $f_{12}=f_{1}+f_{2} . A_{\mathrm{w}}\left(f_{1}, f_{2}, \tau\right)$ and $\Phi_{\mathrm{w}}\left(f_{1}, f_{2}, \tau\right)$ are the instantaneous wavelet biamplitude and biphase. The calculation is done after energy normalization and mean subtraction and the coefficients are computed in the region $\Omega \in\left\{f_{1}+f_{2} \leq\right.$ $\left.f_{s} / 2\right\}$ [35], $f_{s}$ being the sampling frequency. The integration is done over a finite time interval $T: \tau_{0} \leq \tau \leq \tau_{1}$. CWT coefficients of $x(t)$ (likewise of $y(t), z(t)$ ) are given by:

$$
\begin{aligned}
W_{x}(f, \tau) & =\frac{1}{\sqrt{f_{c} / f}} \int_{-\infty}^{\infty} x(t) \psi^{*}\left(\frac{t-\tau}{f_{c} / f}\right) \mathrm{d} t \\
& =\mathcal{A}_{x}(f, \tau) e^{j \phi_{x}(f, \tau)}
\end{aligned}
$$


where $\psi(t)$ is the mother wavelet translated by a factor $\tau$ and scaled by a factor $a, a=f_{c} / f$, wherein $f_{c}$ is the center frequency of the mother wavelet (see (3)). $\mathcal{A}_{x}(f, \tau)$ and $\phi_{x}(f, \tau)$ are the amplitude and phase of CWT, respectively. In this study the complex Morlet wavelet is used, due to its good time localization property, which is given by [40]:

$$
\psi(t)=\frac{1}{\sqrt{\pi \sigma}} e^{-t^{2} / \sigma} e^{j 2 \pi f_{c} t}
$$

where $\sigma$ is the variance parameter. The center frequency of the wavelet $f_{c}$ is set to half the sampling rate $(2 \mathrm{~Hz}$ in this study); its bandwidth is controlled by the parameter $\sigma$, which is set to $1 \mathrm{~Hz}$ in this study.

Let us consider the signal $x(t)=A e^{j\left(2 \pi f_{x} t+\phi\right)}$ without any additive noise for simplification reasons. By doing the variable change $t^{\prime}=\frac{t-\tau}{f_{c} / f}$ in (2) we obtain:

$$
\begin{aligned}
W_{x}(f, \tau) & =\frac{A e^{j \phi}}{\sqrt{f_{c} / f}} \int_{-\infty}^{\infty} e^{j 2 \pi f_{x}\left(t^{\prime} f_{c} / f+\tau\right)} \psi^{*}\left(t^{\prime}\right) \mathrm{d} t^{\prime}\left(\frac{f_{c}}{f}\right) \\
& =\sqrt{\frac{f_{c}}{f}} A e^{j\left(2 \pi f_{x} \tau+\phi\right)} \int_{-\infty}^{\infty} \psi^{*}\left(t^{\prime}\right) e^{j 2 \pi \frac{f_{x} f_{c}}{f} t^{\prime}} \mathrm{d} t^{\prime} \\
& =\sqrt{\frac{f_{c}}{f}} A e^{j\left(2 \pi f_{x} \tau+\phi\right)} e^{-\pi^{2} \sigma f_{c}^{2}\left(f_{x} / f-1\right)^{2}}
\end{aligned}
$$

where $e^{-\pi^{2} \sigma\left(f-f_{c}\right)^{2}}$ is the Fourier transform of the complex mother wavelet $\psi(t)$. Given $x(t), y(t)$ and $z(t)$, the instantaneous wavelet biphase for a triplet of oscillations at frequencies $\left(f_{1}, f_{2}, f_{1}+f_{2}\right)$, based on the above, could be written as:

$$
\begin{gathered}
\Phi_{\mathrm{w}}\left(f_{1}, f_{2}, \tau\right)=\phi_{x}\left(f_{1}, \tau\right)+\phi_{y}\left(f_{2}, \tau\right)-\phi_{z}\left(f_{1}+f_{2}, \tau\right) \\
=2 \pi f_{1} \tau+2 \pi f_{2} \tau-2 \pi\left(f_{1}+f_{2}\right) \tau \\
+\phi_{1}+\phi_{2}-\phi_{12}
\end{gathered}
$$

where $\phi_{1}, \phi_{2}, \phi_{12}$ are the initial phases of the components $f_{1}$, $f_{2}, f_{1}+f_{2}$, respectively. When QPC is present $\phi_{12}=\phi_{1}+\phi_{2}$ (see Fig. 1) and thus $\Phi_{\mathrm{w}}\left(f_{1}, f_{2}, \tau\right)=0$.

QPC detection based on WBS can be blurred by the secondorder properties of the signal [41]. Thus, the normalized WBS, known as squared wavelet bicoherence (WB) is defined as [35]:

$$
b^{2}\left(f_{1}, f_{2}\right)=\frac{\left|B\left(f_{1}, f_{2}\right)\right|^{2}}{B_{\mathrm{N}}\left(f_{1}, f_{2}\right)}
$$

where the dependence of $B\left(f_{1}, f_{2}\right)$ on the amplitude of interacting oscillations is removed by the normalization term:

$$
B_{\mathrm{N}}\left(f_{1}, f_{2}\right)=\int_{T}\left|W_{x}\left(f_{1}, \tau\right) W_{y}\left(f_{2}, \tau\right)\right|^{2} \mathrm{~d} \tau \int_{T}\left|W_{z}\left(f_{12}, \tau\right)\right|^{2} \mathrm{~d} \tau(7)
$$

As a consequence, $0 \leq b^{2} \leq 1$. However, QPC detection by $\mathrm{WB}$ in (6) still presents with some limitations [37]. WB will be close to 1 when wavelet amplitudes $\mathcal{A}_{x}\left(f_{1}, \tau\right), \mathcal{A}_{y}\left(f_{2}, \tau\right)$ and $\mathcal{A}_{z}\left(f_{1}+f_{2}, \tau\right)$ are nonzero and the wavelet biphase relationship $\Phi_{\mathrm{W}}\left(f_{1}, f_{2}, \tau\right)$ is constant across the integration time, which not necessarily implies QPC. In fact, this is a QPU case where the presence of oscillations triplets $\left(f_{1}, f_{2}, f_{1}+f_{2}\right)$ without quadratic phase coupling $\left(\phi_{z}\left(f_{1}+f_{2}, \tau\right) \neq \phi_{x}\left(f_{1}, \tau\right)+\phi_{y}\left(f_{2}, \tau\right)\right)$ will provide high WB values given the constant phase relationships [42]. The QPC detection requires a zero biphase $\left(\phi_{z}\left(f_{1}+f_{2}, \tau\right)=\phi_{x}\left(f_{1}, \tau\right)+\phi_{y}\left(f_{2}, \tau\right)\right)[43]$.

Besides the classical WB, biphase randomization wavelet bicoherence (BRWB) method has been proposed [38]. An ensemble average biphase randomization algorithm was established for bispectrum estimation by adding a biphase randomization term $e^{j \alpha_{k} \Phi_{\mathrm{B}}\left(f_{1}, f_{2}\right)}$ wherein the biphase is multiplied by a random variable, $\alpha_{k}$, which is uniformly distributed within $(-\pi, \pi]$. The randomized WBS is obtained as:

$$
\begin{array}{r}
B_{\mathrm{R}}\left(f_{1}, f_{2}\right)=E\left[\int_{T} W_{x}\left(f_{1}, \tau\right) W_{y}\left(f_{2}, \tau\right) W_{z}^{*}\left(f_{12}, \tau\right)\right. \\
\left.\cdot e^{j \alpha_{k} \Phi_{\mathrm{B}}\left(f_{1}, f_{2}\right)} \mathrm{d} \tau\right]
\end{array}
$$

where $E[$.$] denotes an ensemble average operator for K$ realizations, $\alpha_{k}$, of biphase randomized bispectra. After normalization, the squared BRWB is defined as:

$$
b_{\mathrm{R}}^{2}\left(f_{1}, f_{2}\right)=\frac{\left|B_{\mathrm{R}}\left(f_{1}, f_{2}\right)\right|^{2}}{B_{\mathrm{N}}\left(f_{1}, f_{2}\right)}
$$

When the biphase $\Phi_{\mathrm{B}}\left(f_{1}, f_{2}\right)$ is close to 0 , as in the case of QPC, multiplying the biphase of each realization by a random variable $\alpha_{k}$ will keep the resulting biphase of (8) close to 0 so that a high bicoherence value will remain. A biphase not close to 0 but still constant across the segments (QPU) would generate a high bicoherence $\left(b^{2}\left(f_{1}, f_{2}\right)\right)$ without the presence of QPC. However, when the biphase $\Phi_{\mathrm{B}}\left(f_{1}, f_{2}\right)$ is randomized it results in a reduced bicoherence $\left(b_{\mathrm{R}}^{2}\left(f_{1}, f_{2}\right)\right)$ as a result of expectation of a phasor moving in $(-\pi, \pi]$ [38].

\section{B. Real Wavelet Biphase (RWB)}

In this study a QPC detector, based on wavelet biphase, is proposed. It is suggested that the wavelet biphase carries the information about the nature of quadratic coupling.

Our interest consists in the "phase locking" (zero biphase i.e., $\left.\Phi_{\mathrm{w}}\left(f_{1}, f_{2}, \tau\right)=0\right)$ that represents the perfect QPC, while other values (positive or negative) correspond to the deflection from it. When the frequency $f_{1}+f_{2}$ is absent (Linear case), $\phi_{z}\left(f_{1}+f_{2}, \tau\right)$ does not follow the changes of the other phase components $\phi_{x}\left(f_{1}, \tau\right), \phi_{y}\left(f_{2}, \tau\right)$ leading to an instantaneous wavelet biphase far away from a constant zero value ("phase locking"). When there are two components, with frequencies $f_{1}, f_{2}$ and phases $\phi_{x}\left(f_{1}, \tau\right)$, $\phi_{y}\left(f_{2}, \tau\right)$ respectively, as well as an oscillation at frequency $f_{1}+f_{2}$ with phase $\phi_{z}\left(f_{1}+f_{2}, \tau\right)$, QPC or QPU can be present depending on the phases $\phi_{z}\left(f_{1}+f_{2}, \tau\right), \phi_{x}\left(f_{1}, \tau\right)$ and $\phi_{y}\left(f_{2}, \tau\right)$ along the time. If $\phi_{z}\left(f_{1}+f_{2}, \tau\right)$ is the sum of the phases $\phi_{x}\left(f_{1}, \tau\right), \phi_{y}\left(f_{2}, \tau\right)$ for the whole analyzed interval, QPC is present since $\Phi_{\mathrm{W}}\left(f_{1}, f_{2}, \tau\right)=0$. In QPU this specific phase relationship that leads to the "phase locking" is absent. The phase $\phi_{z}\left(f_{1}+f_{2}, \tau\right)$ is not the sum of the other phase components $\left(\Phi_{\mathrm{w}}\left(f_{1}, f_{2}, \tau\right) \neq 0\right)$ but a different value providing a non-zero instantaneous wavelet biphase that represents the absence of higher order phase relationship. 
Based on the aforementioned approach, the mathematical formula of (1) is simplified in terms of the complex argument removing directly the amplitude effect:

$$
B_{\Phi}\left(f_{1}, f_{2}\right)=\int_{T} e^{j \Phi_{\mathrm{W}}\left(f_{1}, f_{2}, \tau\right)} \mathrm{d} \tau
$$

Real Wavelet Biphase is defined from the real part of (10) with a time normalization term as:

$$
\begin{aligned}
b_{\Phi}\left(f_{1}, f_{2}\right) & =\frac{1}{T} \Re\left\{B_{\Phi}\left(f_{1}, f_{2}\right)\right\} \\
& =\frac{1}{T} \int_{T} \cos \left(\Phi_{\mathrm{W}}\left(f_{1}, f_{2}, \tau\right)\right) \mathrm{d} \tau
\end{aligned}
$$

This simple computation is the mean cosine value of the instantaneous wavelet biphase in the interval $T$ and is bounded between -1 and 1 . Values close to 1 correspond to perfect QPC. In order to compare the computed amount of QPC with the other methods, the squared values of RWB $\left(b_{\Phi}^{2}\left(f_{1}, f_{2}\right)\right)$ are considered:

$$
b_{\Phi}^{2}\left(f_{1}, f_{2}\right)=\left\{\begin{aligned}
0, & b_{\Phi}\left(f_{1}, f_{2}\right)<N_{\mathrm{TH}}\left(f_{1}, f_{2}\right) \\
b_{\Phi}^{2}\left(f_{1}, f_{2}\right), & b_{\Phi}\left(f_{1}, f_{2}\right) \geq N_{\mathrm{TH}}\left(f_{1}, f_{2}\right)
\end{aligned}\right.
$$

where the lowest value of $N_{\mathrm{TH}}\left(f_{1}, f_{2}\right)$ is given by the upper bound of the statistical noise that the replacement of the integrals by summations and the use of non-orthogonal mother wavelet introduce in the computation of all wavelet bispectral based measures [35]:

$$
N_{\text {TH }}\left(f_{1}, f_{2}\right) \geq\left[\frac{f_{s}}{\min \left(\left|f_{1}\right|,\left|f_{2}\right|,\left|f_{1}+f_{2}\right|\right) \cdot 2 N}\right]^{\frac{1}{2}}
$$

The detection of short-lived couplings demands the computation to be done in small intervals which, contrariwise, would reduce the resolution, causing errors in phase estimation. For a time-varying tracking of QPC the definition of instantaneous bispectra is given by:

$$
\begin{gathered}
B\left(f_{1}, f_{2}, t\right)=\int_{t-T^{\prime} / 2}^{t+T^{\prime} / 2} A_{\mathrm{W}}\left(f_{1}, f_{2}, \tau\right) e^{j \Phi_{\mathrm{W}}\left(f_{1}, f_{2}, \tau\right)} \mathrm{d} \tau \\
B_{\mathrm{N}}\left(f_{1}, f_{2}, t\right)=\int_{t-T^{\prime} / 2}^{t+T^{\prime} / 2}\left|W_{x}\left(f_{1}, \tau\right) W_{y}\left(f_{2}, \tau\right)\right|^{2} \mathrm{~d} \tau \int_{t-T^{\prime} / 2}^{t+T^{\prime} / 2}\left|W_{z}\left(f_{12}, \tau\right)\right|^{2} \mathrm{~d} \tau \\
B_{\Phi}\left(f_{1}, f_{2}, t\right)=\frac{1}{T^{\prime}} \int_{t-T^{\prime} / 2}^{t+T^{\prime} / 2} e^{j \Phi_{\mathrm{W}}\left(f_{1}, f_{2}, \tau\right)} \mathrm{d} \tau
\end{gathered}
$$

where CWT computation is done in a window of length $T$ centered at the desired time point $t$, while the integration is carried out for the $T^{\prime}(<T)$ central seconds. Then, the definition of instantaneous WB $\left(b^{2}\left(f_{1}, f_{2}, t\right)\right)$, instantaneous $\operatorname{BRWB}\left(b_{\mathrm{R}}^{2}\left(f_{1}, f_{2}, t\right)\right)$ and instantaneous RWB $\left(b_{\Phi}^{2}\left(f_{1}, f_{2}, t\right)\right)$, which allow the detection of couplings with at least duration $T^{\prime}$, can be done based on (14), (15) and (16). $N_{\text {тн }}\left(f_{1}, f_{2}, t\right)$ can be defined for each time instant $t$ and the values in $b^{2}\left(f_{1}, f_{2}, t\right), b_{\mathrm{R}}^{2}\left(f_{1}, f_{2}, t\right)$ and $b_{\Phi}^{2}\left(f_{1}, f_{2}, t\right)$ that do not exceed it are set to zero. The final value of the time-varying threshold $N_{\text {TH }}\left(f_{1}, f_{2}, t\right)$ will be defined based on surrogate data analysis and will be explained in the next section.

\section{Surrogate Data Analysis}

To obtain a statistically significant estimation of QPC, a surrogate method is employed. In [44], an approach was described, which takes the Fourier transform of the original data and then assigns a random phase to each frequency component, resulting in time series with the same power spectrum as the original data, but which are randomized in every other respect. Regarding HOS-surrogates, the surrogate data will have the same amplitude distribution $\left(A_{\mathrm{W}}\left(f_{1}, f_{2}, \tau\right)\right)$, as the original data, but any temporal phase relationship $\left(\Phi_{\mathrm{W}}\left(f_{1}, f_{2}, \tau\right)\right)$ that may have been in the original data should be destroyed.

A phase-randomized bispectrum for surrogate analysis $\left(B_{\mathrm{S}}\left(f_{1}, f_{2}, t\right)\right)$ has been proposed by Scully et al. [45] replacing the biphase at each time point in the analyzed interval $\left(T^{\prime}\right)$ with a uniform random variable $\theta$ in $U\left[-\pi:-\theta_{\mathrm{th}}, \theta_{\mathrm{th}}: \pi\right]$ :

$$
B_{\mathrm{S}}\left(f_{1}, f_{2}, t\right)=\int_{t-T^{\prime} / 2}^{t+T^{\prime} / 2} A_{\mathrm{W}}\left(f_{1}, f_{2}, \tau\right) e^{j \theta(\tau)} \mathrm{d} \tau
$$

Normalizing with $B_{\mathrm{N}}\left(f_{1}, f_{2}, t\right)$ we obtain the Surrogate Phase Randomized Bicoherence (SPRB):

$$
b_{\mathrm{s}}^{2}\left(f_{1}, f_{2}, t\right)=\frac{\left|B_{\mathrm{S}}\left(f_{1}, f_{2}, t\right)\right|^{2}}{B_{\mathrm{N}}\left(f_{1}, f_{2}, t\right)}
$$

In [45] a specific threshold $\theta_{\text {th }}=1$ was used, while in our study we will examine how different values of $\theta_{\text {th }}$ threshold affect QPC detection not only in case of a perfect coupling but also in case of QPC with a certain delay, as it will be explained later. SPRB is determined for 100 realizations and the mean plus 2 standard deviations is used as threshold $N_{\text {Тн }}\left(f_{1}, f_{2}, t\right)$ at each time point $t$ [45], substituting the value of (13) only when it is greater. Finally, the original values of $b^{2}\left(f_{1}, f_{2}, t\right)$, $b_{\mathrm{R}}^{2}\left(f_{1}, f_{2}, t\right)$ and $b_{\Phi}^{2}\left(f_{1}, f_{2}, t\right)$ greater than this threshold will be preserved, otherwise are set to zero.

\section{Quantification of Nonlinear Cardiorespiratory Coupling}

The complex structure of HRV includes various oscillations that might be nonlinearly related to respiration [7]-[12]. Our hypothesis is that HRV signal might be formed as an output of a quadratic nonlinear system:

$$
x_{\mathrm{QPC}}(t)=x_{\mathrm{L}}(t)+x_{\mathrm{R}}(t)+\frac{\varepsilon_{\mathrm{L}}}{\varepsilon_{\mathrm{L}, \mathrm{R}}} x_{\mathrm{L}}(t) x_{\mathrm{R}}(t)+\frac{\varepsilon_{\mathrm{R}}}{\varepsilon_{\mathrm{R}, \mathrm{R}}} x_{\mathrm{R}}^{2}(t)
$$

wherein $x_{\mathrm{L}}(t)$ is a low frequency (LF) oscillation, in the range from 0.04 to $0.15 \mathrm{~Hz}$, that represents a component of sympathetic and parasympathetic activation and $x_{\mathrm{R}}(t)$ is a high frequency (HF) component, usually in the range from 0.15 to $0.4 \mathrm{~Hz}$, related to the respiration (vagal activity) that controls the HRV not only through a linear function but also with nonlinear ones. Interactions of vagal-mediated and sympathetic-related components of HRV $\left(x_{\mathrm{L}}(t) x_{\mathrm{R}}(t), x_{\mathrm{R}}^{2}(t)\right)$ might be a way to regulate heart rate and blood pressure, since intrinsic cardiac nervous system integrates mechanosensitive and chemosensitive neuron inputs with efferent information from both the sympathetic and parasympathetic inputs from the brain [46]. The coupling strengths $\varepsilon_{\mathrm{L}}$ and $\varepsilon_{\mathrm{R}}$ could be different, while $\varepsilon_{\mathrm{L}, \mathrm{R}}=\sigma_{\mathrm{L}, \mathrm{R}}^{2} / \sigma_{\mathrm{R}}^{2}$ and $\varepsilon_{\mathrm{R}, \mathrm{R}}=\sigma_{\mathrm{R}, \mathrm{R}}^{2} / \sigma_{\mathrm{R}}^{2}$ are two 
normalization terms based on the power of each nonlinear component $\left(\sigma_{\mathrm{L}, \mathrm{R}}^{2}, \sigma_{\mathrm{R}, \mathrm{R}}^{2}\right)$ with respect to respiration power $\left(\sigma_{\mathrm{R}}^{2}\right)$.

Based on the above definition, the most adequate RWB approach to investigate cross-phase relationships and timevariant nonlinear cardiorespiratory couplings is the instantaneous cross-RWB $\left(b_{\Phi}^{2}\left(f_{1}, f_{2}, t\right)\right)$. The signals $x(t)$ and $z(t)$ correspond to HRV, while $y(t)$ to respiration. Thus, $\Phi_{\mathrm{w}}\left(f_{1}, f_{2}, \tau\right)$ in (5) is modified as:

$$
\Phi_{\mathrm{w}}\left(f_{1}, f_{2}, \tau\right)=\phi_{x}\left(f_{1}, \tau\right)+\phi_{y}\left(f_{2}, \tau\right)-\phi_{x}\left(f_{1}+f_{2}, \tau\right)
$$

Regarding any HRV analysis there is a limitation that should be taken into account. The intrinsic sampling frequency of $\mathrm{HRV}$ is given by the heart rate $f_{\mathrm{HR}}$. As a result, the maximum frequency with physiological meaning, which can be analyzed is defined as half the mean $\mathrm{HR} f_{1}+f_{2}<f_{\mathrm{HR}} / 2$ in the analysis window [47]. This frequency is much lower than $f_{s} / 2$ and so more restrictive than the presented in section II.A. Spectral components above this frequency are due to spectrum repetitions and thus, values in bi-frequency domain, whose coordinates exceed $f_{\mathrm{HR}} / 2$ were set to zero.

Feature Set: Respiratory frequency estimation is included in HRV analysis redefining the HF band centered at respiratory frequency [48], [49]. A quadratic phase coupling between respiration and LF or HF HRV components will be identified based on the respiratory rate information $\left(f_{r}(t)\right)$, which is computed in each segment by:

$$
f_{r}(t)=\underset{f}{\arg \max }\left(\int_{t-T^{\prime} / 2}^{t+T^{\prime} / 2}\left|W_{y}(f, \tau)\right| d \tau\right)
$$

Once the respiratory frequency has been identified, a frequency range around the dominant rate in each time instant $t$ is defined as $\Omega_{\mathrm{R}}=\left[f_{r}(t)-0.05, f_{r}(t)+0.05\right] \mathrm{Hz}$. For HRV signal, two ranges are considered, $\Omega_{\mathrm{R}}$ for the RSA component at HF band and $\Omega_{\mathrm{L}}=\left[0.04, f_{r}(t)-0.05\right] \mathrm{Hz}$, to take into account also the sympathetic activity at LF band. Based on the previous ranges, two regions are defined where QPC will be assessed, $\Omega_{\mathrm{L}, \mathrm{R}}:\left\{f_{1} \in \Omega_{\mathrm{L}}, f_{2} \in \Omega_{\mathrm{R}}\right\}$ and $\Omega_{\mathrm{R}, \mathrm{R}}:\left\{f_{1} \in \Omega_{\mathrm{R}}, f_{2} \in \Omega_{\mathrm{R}}\right\}$. Recall from (20), $f_{1}$ refers to HRV $(x(t))$ while $f_{2}$ to respiration $(y(t))$. Real respiratory signals (Fig. 2) may contain other components besides the dominant one, which would generate peaks in other regions in the bi-frequency domain, although they are not considered in this study. The following parameters are introduced to detect QPC in the regions of interest:

$$
C_{\mathcal{I}}^{\Phi}(t)=\max _{f_{1}, f_{2} \in \Omega_{\mathcal{I}}}\left\{b_{\Phi}^{2}\left(f_{1}, f_{2}, t\right)\right\}
$$

where $\mathcal{I} \in\{[L, R],[R, R]\}$ refers to the region $\Omega_{\mathcal{I}}$ where the parameter was computed. If the region of interest is the whole bi-frequency domain $\Omega$ then the subscript $\mathcal{I}$ is omitted $\left(C^{\Phi}(t)\right)$. The detection is done based on RWB method $\left(b_{\Phi}^{2}\left(f_{1}, f_{2}, t\right)\right)$. For comparison purposes, QPC detection can be done based on (22) for WB $\left(C_{\mathcal{I}}(t)\right)$ replacing $b_{\Phi}^{2}\left(f_{1}, f_{2}, t\right)$ with $b^{2}\left(f_{1}, f_{2}, t\right)$, while for BRWB $\left(C_{\mathcal{I}}^{\mathrm{R}}(t)\right)$ with $b_{\mathrm{R}}^{2}\left(f_{1}, f_{2}, t\right)$. $C_{\mathcal{I}}(t), C_{\mathcal{I}}^{\mathrm{R}}(t)$ and $C_{\mathcal{I}}^{\Phi}(t)$ are indexes of phase synchronization since get lower values when there is a deviation from the quadratic phase locking. However, as it will be shown later, none of them can be used to quantify QPC strength.
TABLE I

FEATURES USED IN THIS STUDY

\begin{tabular}{cc}
\hline \hline Feature & Meaning \\
\hline $\bar{f}_{\mathrm{r}}$ & Mean rate of respiration signal $(y(t))$ \\
$\bar{E}_{\mathrm{R}, \mathrm{R}}$ & Mean NWB between HRV, $x(t)$, at frequencies \\
$\bar{E}_{\mathrm{L}, \mathrm{R}}$ & around $f_{r}$ and respiration, $y(t)\left(\Omega_{\mathrm{R}, \mathrm{R}}\right)$ \\
& Mean NWB between HRV, $x(t)$, at frequencies \\
& lower than $f_{r}$ and respiration, $y(t)\left(\Omega_{\mathrm{L}, \mathrm{R}}\right)$ \\
$T_{\mathrm{R}, \mathrm{R}}$ & Time percentage of QPC in $\Omega_{\mathrm{R}, \mathrm{R}}$ \\
$T_{\mathrm{L}, \mathrm{R}}$ & Time percentage of QPC in $\Omega_{\mathrm{L}, \mathrm{R}}$ \\
\hline \hline
\end{tabular}

Once the detection of QPC is performed based on $N_{\text {тн }}\left(f_{1}, f_{2}, t\right)$ and is positive, i.e. $C_{\mathcal{I}}^{\Phi}(t)>0$, it makes sense to quantify the coupling strength at the involving frequencies $\hat{f}_{1}, \hat{f}_{2}(23)$. Note that these frequencies depend on $t$, but this dependence has been omitted from the notation for simplicity. In order to quantify QPC we proposed the Normalized Wavelet Biamplitude (NWB) (24):

$$
\begin{gathered}
\left(\hat{f}_{1}, \hat{f}_{2}\right)=\underset{f_{1}, f_{2} \in \Omega_{\mathcal{I}}}{\arg \max }\left\{b_{\Phi}^{2}\left(f_{1}, f_{2}, t\right)\right\} \\
E_{\mathcal{I}}(t)=\frac{\left|B\left(\hat{f}_{1}, \hat{f}_{2}, t\right)\right|}{\int_{t-T^{\prime} / 2}^{t+T^{\prime} / 2}\left|W_{x}\left(\hat{f}_{1}, \tau\right) W_{y}\left(\hat{f}_{2}, \tau\right)\right| \mathrm{d} \tau}
\end{gathered}
$$

where the influence of the interacting oscillations amplitudes through $\left(W_{x}\left(f_{1}, \tau\right)\right)$ and $\left(W_{y}\left(f_{2}, \tau\right)\right)$ on $\left|B\left(f_{1}, f_{2}, t\right)\right|$ is removed. NWB is influenced only by the coupling strength and the nonlinear oscillations amplitude in $\operatorname{HRV}\left(\left|W_{x}\left(f_{1}+f_{2}, \tau\right)\right|\right)$. Moreover, if the phase is not maintained constant at the integration interval, $E_{\mathcal{I}}(t)$ will get lower values. Note that QPC detection can be carried out through the normalized domains $b^{2}\left(f_{1}, f_{2}, t\right), b_{\mathrm{R}}^{2}\left(f_{1}, f_{2}, t\right)$ or $b_{\Phi}^{2}\left(f_{1}, f_{2}, t\right)$, while its quantification is done always through WBS $\left(B\left(f_{1}, f_{2}, t\right)\right)$.

The feature set, used in the present study and summarized in Table I, consists of the following parameters: (i) the mean respiratory rate $\left(\bar{f}_{r}\right)$ and, for the regions of interest $\Omega_{\mathrm{L}, \mathrm{R}}$ and $\Omega_{\mathrm{R}, \mathrm{R}}$, (ii) the temporal average of NWB values $\left(\bar{E}_{\mathcal{I}}\right)$, and (iii) the time percentage $T_{\mathcal{I}}$ in which QPC was present $\left(C_{\mathcal{I}}^{\Phi}(t)>0\right)$.

\section{E. Measurements}

Seventeen volunteers (age $28.5 \pm 2.8$ years, 11 males) underwent a head-up tilt table test recorded at University of Zaragoza. The protocol consisted of: 4 minutes in early supine position $\left(T_{e}\right), 5$ minutes head-up tilted to an angle of $70^{\circ}$ $\left(T_{h}\right)$ and 4 minutes back to later supine position $\left(T_{l}\right)$. The standard 12-lead ECG and respiratory signals were recorded using the BIOPAC MP 150 system with a sampling frequency of $1 \mathrm{kHz}$ and $125 \mathrm{~Hz}$ respectively. The respiratory signal was recorded through a strain gauge transducer. HRV was generated from the beat occurrence time series, detected on lead V4 of the ECG, based on the integral pulse frequency modulation (IPFM) model, which accounts for the presence of ectopic beats [50] and sampled at a sampling frequency $\left(f_{s}\right)$ of $4 \mathrm{~Hz}$. The respiration signal was downsampled to 4 


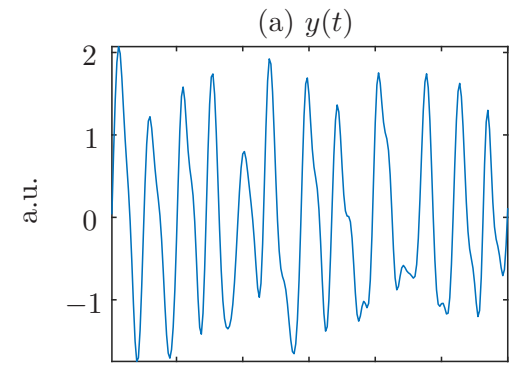

$\begin{array}{llllll}10 & 20 & 30 & 40 & 50 & 60\end{array}$

(d) $\left|W_{y}(f, t)\right|$

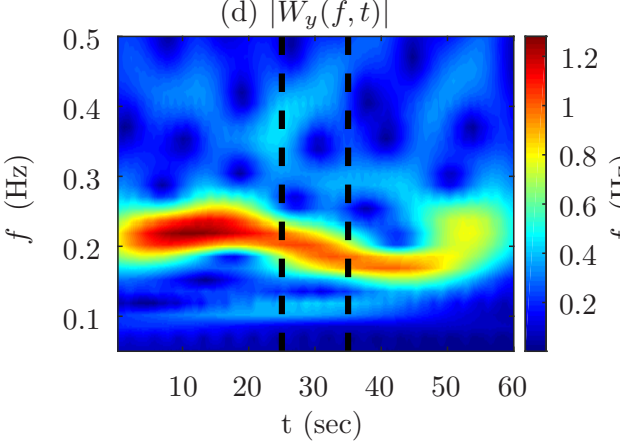

(b) $x(t)$

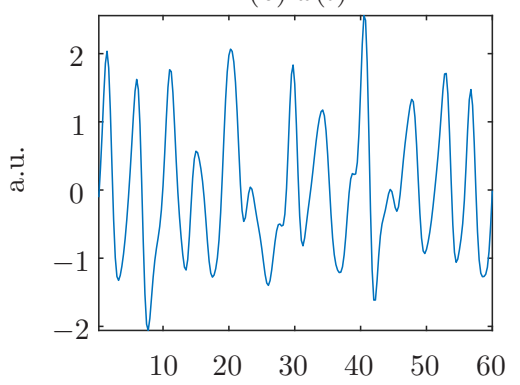

(e) $\left|W_{x}(f, t)\right|$

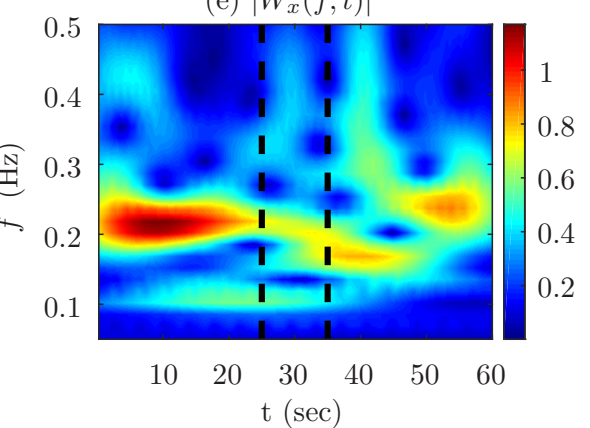

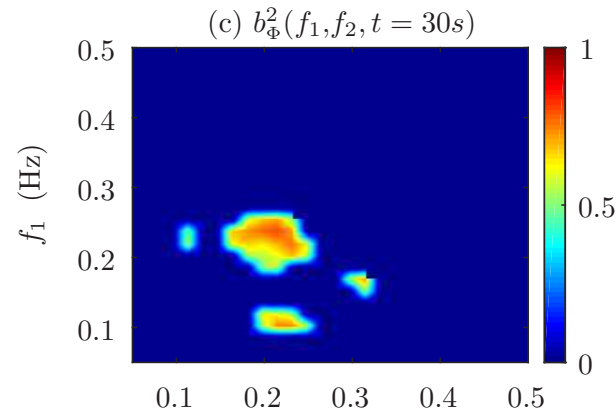

(f) $\left|B\left(f_{1}, f_{2}, t=30 s\right)\right|$

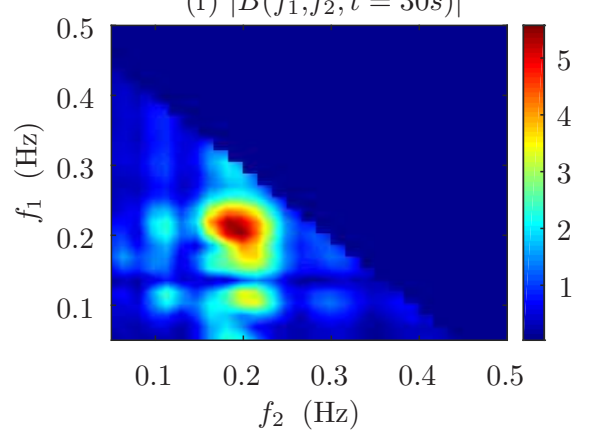

Fig. 2. (a) Respiration signal $y(t)$, (b) HRV signal $x(t)$, (c) Instantaneous cross-RWB $\left(b_{\Phi}^{2}\left(f_{1}, f_{2}, t\right)\right)$ for $t=30 s$, (d) CWT amplitude of $y(t)$, (e) CWT amplitude of $x(t)$ and (f) Amplitude of instantaneous WBS $\left(\left|B\left(f_{1}, f_{2}, t\right)\right|\right)$ for $t=30 s$. Bold dashed lines indicate the integration time interval $T^{\prime}=10 s$.

Hz. Both HRV and respiration were filtered, with a band-pass filter (Butterworth 6th order with low/high cutoff frequencies of 0.04 and $0.8 \mathrm{~Hz}$, respectively).

\section{F. Simulation Study}

A simulation was carried out to evaluate the performance of WB, BRWB and RWB in assessing QPC. It offers the ability to investigate the correct detection of QPC and its reliability in cases of linear and QPU. Based on the model described in (19), the two principal components reenacting the sympathetic $\left(x_{\mathrm{L}}(t)\right)$ and parasympathetic $\left(x_{\mathrm{R}}(t)\right)$ activity would be represented with oscillations around the frequencies $f_{\mathrm{L}}=0.1 \mathrm{~Hz}$ and $f_{\mathrm{R}}=0.25 \mathrm{~Hz}$ (a common respiratory frequency), respectively.

To do that, two white gaussian noises are filtered using two autoregressive models, which are designed locating at the polar plane two conjugated poles at low frequency for sympathetic activity $\left(p_{1}^{\mathrm{L}}=0.95 e^{j 2 \pi f_{\mathrm{L}} / f_{s}}, p_{2}^{\mathrm{L}}=p_{1}^{\mathrm{L} *}\right)$ and at high frequency simulating RSA $\left(p_{1}^{\mathrm{R}}=0.95 e^{j 2 \pi f_{\mathrm{R}} / f_{s}}, p_{2}^{\mathrm{R}}=p_{1}^{\mathrm{R} *}\right)$ [51]. The two signals $x_{\mathrm{L}}(t), x_{\mathrm{R}}(t)$ are normalized to have the same power and the respiration signal is defined as $y(t)=x_{\mathrm{R}}(t)$.

In earlier works [52], [53], it has been suggested that the nonlinear phase coupling occurs when the systems are in phase $(\mathrm{QPC})$, or with a constant delay $\left(\mathrm{QPC}_{\mathrm{D}}\right)$. In order to study the effect of a constant delay between components we propose a modification of (19):

$$
\begin{aligned}
x_{\mathrm{D}}(t)=x_{\mathrm{L}}(t)+x_{\mathrm{R}}(t) & +\frac{\varepsilon_{\mathrm{L}}}{\varepsilon_{\mathrm{L}, \mathrm{R}}} x_{\mathrm{L}}\left(t-t_{\mathrm{L}}\right) x_{\mathrm{R}}\left(t-t_{\mathrm{R}}\right) \\
& +\frac{\varepsilon_{\mathrm{R}}}{\varepsilon_{\mathrm{R}, \mathrm{R}}} x_{\mathrm{R}}^{2}\left(t-t_{\mathrm{R}}\right)
\end{aligned}
$$

where $t_{\mathrm{L}}=\phi_{\mathrm{D}} /\left(2 \pi f_{\mathrm{L}}\right), t_{\mathrm{R}}=\phi_{\mathrm{D}} /\left(2 \pi f_{\mathrm{R}}\right)$ are the delay parameters, which are assumed for simplicity based on common phase shift value $\phi_{\mathrm{D}}$. To simulate QPU, nonlinear components $x_{\mathrm{L}}(t) x_{\mathrm{R}}(t), x_{\mathrm{R}}^{2}(t)$ are phase-randomized:

$$
\begin{aligned}
x_{\mathrm{QPU}}(t)=x_{\mathrm{L}}(t)+x_{\mathrm{R}}(t) & +\frac{\varepsilon_{\mathrm{L}}}{\varepsilon_{\mathrm{L}, \mathrm{R}}} \mathcal{F}^{-1}\left\{\left|F_{\mathrm{L}, \mathrm{R}}(f)\right| e^{j \phi_{\mathrm{L}, \mathrm{R}}}\right\} \\
& +\frac{\varepsilon_{\mathrm{R}}}{\varepsilon_{\mathrm{R}, \mathrm{R}}} \mathcal{F}^{-1}\left\{\left|F_{\mathrm{R}, \mathrm{R}}(f)\right| e^{j \phi_{\mathrm{R}, \mathrm{R}}}\right\}
\end{aligned}
$$

where $\mathcal{F}^{-1}$ denotes the inverse Fourier transform, $F_{\mathrm{L}, \mathrm{R}}(f)$ the Fourier Transform of $x_{\mathrm{L}}(t) x_{\mathrm{R}}(t), F_{\mathrm{R}, \mathrm{R}}(f)$ the Fourier Transform of $x_{\mathrm{R}}^{2}(t)$, and $\phi_{\mathrm{L}, \mathrm{R}}, \phi_{\mathrm{R}, \mathrm{R}}$, two random variables uniformly distributed within $(-\pi, \pi]$. As a result, the zero biphase relationship of nonlinear components is destroyed but their spectral properties remain.

The sampling frequency $f_{s}$ is $4 \mathrm{~Hz}$ to be comparable with the $f_{s}$ of the real signals. In our approach we are interested in the estimation of short-live couplings and thus, the integration time will be set to $T^{\prime}=10 \mathrm{~s}$ centered at the window of length $T=60 \mathrm{~s}$ for all the methods. In BRWB 100 realizations $(K=$ 100) have been done for the randomized biphase procedure.

1) Reliability of QPC detection: The first simulation is an attempt to ascertain the reliability of QPC detection $\left(C_{\mathcal{I}}^{\Phi}(t)\right)$ considering the delay influence $\left(\phi_{\mathrm{D}}\right)$ in $(25)$ and to show how the choice of $\theta_{\text {th }}$ affects the surrogate derived thresholds. Phase deviations from the ideal quadratic ones will be simulated for a low strength coupling $\left(\varepsilon_{\mathrm{L}}=\varepsilon_{\mathrm{R}}=0.1\right)$. The purpose of this analysis is to prove the ability of each algorithm to provide information regarding the deflection from the specific coupling named QPC in a controlled manner. The phase shift $\phi_{\mathrm{D}}$ varies from 0 to $1.57 \mathrm{rad}$ with a step of $0.31 \mathrm{rad}$ to simulate a progressive deviation from the perfect "phase locking", while the threshold $\theta_{\text {th }}$ for surrogate data varies from 0 to 1.25 rad with a step of $0.25 \mathrm{rad}$. The simulation is repeated 100 times for each case and the median values are presented for 
TABLE II

THE PARAMETER SET OF TIME-VARYING QPC SIMULATION

\begin{tabular}{cccc}
\hline \hline Intervals (sec) & Duration (sec) & Coupling & Parameters \\
\hline $0-60$ & 60 & QPU & $\varepsilon_{\mathrm{L}}=0, \varepsilon_{\mathrm{R}}=0.5$ \\
$60-85$ & 25 & Linear & $\varepsilon_{\mathrm{L}}=\varepsilon_{\mathrm{R}}=0$ \\
$85-105$ & 20 & QPC & $\varepsilon_{\mathrm{L}}=0, \varepsilon_{\mathrm{R}}=0 \rightarrow 0.5$ \\
$105-125$ & 20 & QPC & $\varepsilon_{\mathrm{L}}=0, \varepsilon_{\mathrm{R}}=0.5 \rightarrow 0$ \\
$125-145$ & 20 & Linear & $\varepsilon_{\mathrm{L}}=\varepsilon_{\mathrm{R}}=0$ \\
$145-170$ & 25 & QPC & $\varepsilon_{\mathrm{L}}=0.25, \varepsilon_{\mathrm{R}}=0$ \\
$170-185$ & 15 & Linear & $\varepsilon_{\mathrm{L}}=\varepsilon_{\mathrm{R}}=0$ \\
$185-215$ & 30 & QPC & $\varepsilon_{\mathrm{L}}=0, \varepsilon_{\mathrm{R}}=0.1$ \\
$215-235$ & 20 & Linear & $\varepsilon_{\mathrm{L}}=\varepsilon_{\mathrm{R}}=0$ \\
$235-280$ & 45 & QPC & $\varepsilon_{\mathrm{L}}=0.5, \varepsilon_{\mathrm{R}}=0$ \\
$280-295$ & 15 & Linear & $\varepsilon_{\mathrm{L}}=\varepsilon_{\mathrm{R}}=0$ \\
$295-335$ & 40 & QPC & $\varepsilon_{\mathrm{L}}=0, \varepsilon_{\mathrm{R}}=0.5$ \\
$335-370$ & 35 & Linear & $\varepsilon_{\mathrm{L}}=\varepsilon_{\mathrm{R}}=0$ \\
\hline \hline
\end{tabular}

comparison. Results will be compared to the ones obtained by WB and BRWB.

2) Robustness of QPC assessment: The second simulation is an attempt, given the optimized threshold $\theta_{\text {th }}$, to ascertain the robustness of QPC quantification $\left(E_{\mathcal{I}}(t)\right)$ under different coupling strengths and in the presence of linear and QPU relationships. Without considering any delay influence $\left(\phi_{\mathrm{D}}=0\right)$ (19), the linear case of cardiorespiratory couplings is simulated selecting $\varepsilon=\varepsilon_{\mathrm{L}}=\varepsilon_{\mathrm{R}}=0$, while different level of QPC with $\varepsilon=\varepsilon_{\mathrm{L}}=\varepsilon_{\mathrm{R}}$, which varies from 0.2 to 1 with a step of 0.2 . The same strength variations are considered for QPU, which is obtained through (26). The simulation is repeated 100 times and the median values are presented for comparison. Results will be compared to the ones obtained by WB and BRWB.

3) Time-varying QPC tracking: This simulation has been performed in order to investigate the ability of the proposed method to detect and quantify dynamic QPC. First, the components $x_{\mathrm{L}}(t), y_{\mathrm{R}}(t)$ are generated and they will be present during the whole simulation as the linear components of HRV. Then within the duration of the signal there will be segments of QPC (19), QPC with a constant delay (25) and QPU (26). Table II describes the various durations and coupling strengths of the different stages that compose the simulated signal. Note that, in this simulation, one of $\varepsilon_{\mathrm{L}}$ or $\varepsilon_{\mathrm{R}}$ is set to 0 in the coupling intervals in order to investigate RWB $\left(C^{\Phi}(t)\right)$ accuracy to track down the coordinates of a single peak in the whole bi-frequency domain $\Omega$ (not a specific subregion $\Omega_{\mathcal{I}}$ ). For instance, when $\varepsilon_{\mathrm{L}}=0$, and $\varepsilon_{\mathrm{R}} \neq 0$ the expected maximum value is $b_{\Phi}^{2}\left(f_{\mathrm{R}}, f_{\mathrm{R}}, t\right)$, while for $\varepsilon_{\mathrm{L}} \neq 0$ and $\varepsilon_{\mathrm{R}}=0$ is $b_{\Phi}^{2}\left(f_{\mathrm{L}}, f_{\mathrm{R}}, t\right)$. Furthermore, we simulated different QPC scenarios, where the coupling strength was time-varying (85-125 s), constant (145$170 \mathrm{~s}, 185-215 \mathrm{~s}, 235-280 \mathrm{~s}$ ) or in presence of delay (295$335 \mathrm{~s})$. Time-varying coupling is denoted in Table II with an arrow indicating a linear increase or decrease in the coupling strength. For the remaining intervals, the first and last $5 \mathrm{~s}$ of theirs duration are also time-varying couplings phenomena (linear increase/decrease until the desired coupling strength). Regarding $\mathrm{QPC}_{\mathrm{D}}, \phi_{\mathrm{D}}$ is randomly distributed in $[-1.2,1.2] \mathrm{rad}$, while for QPU, $\phi_{\mathrm{L}, \mathrm{R}}$ and $\phi_{\mathrm{R}, \mathrm{R}}$ are randomly distributed within $(-\pi, \pi] \mathrm{rad}$ in each realization. The whole signal is analyzed through overlapped windows of length $T$ with a step factor of 2 seconds. The simulation is repeated 100 times and the median values of $\hat{f}_{1}, \hat{f}_{2}, C^{\Phi}(t)$ and $E(t)$ are presented.

\section{G. Statistical Analysis of Cardiorespiratory Coupling during Tilt Table Test}

The real signals are analyzed through overlapped windows of length $T=60 \mathrm{~s}$ with a step factor of $2 \mathrm{~s}$ and integration interval $T^{\prime}=10 \mathrm{~s}$ and a statistical analysis is performed for the feature set defined in Table I. In order to investigate changes of cardiorespiratory couplings at head-up tilt $T_{h}$ with respect to early supine $T_{e}$ and cardiorespiratory couplings at later supine position $T_{l}$ with respect to $T_{e}$, two statistical tests were carried out separately. A Student's t-test or a Wilcoxon test was implemented for each feature depending on the distribution of the data, Gaussian or not, respectively. All statistical tests were paired for each subject in each comparison $\left(T_{h}\right.$ vs $T_{e}, T_{l}$ vs $\left.T_{e}\right)$ and statistical differences are considered when the null hypothesis was rejected with significance level $p<0.05$. In order to avoid transition phenomena at the beginning and at the end of $T_{h}$ stage, as well as to retain similar number of segments in all the stages only the 4 central minutes of $T_{h}$ phase were considered.

\section{RESUlTS}

\section{A. Simulation Study}

1) Reliability of QPC detection: Fig. 3 shows the feasibility of each method in QPC detection under a delay influence $\left(\phi_{\mathrm{D}}\right)$ and how the choice of $\theta_{\text {th }}$ in surrogate data analysis affects the outcome. Phase deviations from the ideal quadratic ones lead to reduced values for BRWB and RWB, while WB method seems to ignore phase alternations and thus cannot be used to estimate properly QPC. The frequency resolution is worse at lower $\left(\Omega_{\mathrm{L}, \mathrm{R}}\right)$ than at higher frequencies $\left(\Omega_{\mathrm{R}, \mathrm{R}}\right)$ and consequently the maximum phase shift, for which QPC is detected, is lower for $\Omega_{\mathrm{L}, \mathrm{R}}$ than for $\Omega_{\mathrm{R}, \mathrm{R}}$. When $\theta_{\mathrm{th}} \rightarrow \pi$ the phase randomization of surrogate data is not effective, making impossible the detection of a perfect QPC in $\Omega_{\mathrm{L}, \mathrm{R}}$.

Limiting the surrogate biphase away from 0 leads to a less random evolution of the phase $(\theta(\tau))$, which could create constant (non-zero) phase relationships in a small integration interval $T$. The surrogate threshold is a bicoherence value and is sensitive to constant phase relationships [37]. When $\theta_{\text {th }} \rightarrow \pi$, the upper values of surrogate threshold are high. Based on the above, the optimum threshold $\left(\theta_{\mathrm{th}}\right)$ for surrogate data which allows the detection of QPC with the maximum possible delay, is given for $\theta_{\mathrm{th}}=0$, as it can be seen Fig. 3 .

2) Robustness of QPC assessment: Fig. 4 shows the robustness of methods on QPC detection and its strength quantification. BRWB $\left(C_{\mathcal{I}}^{\mathrm{R}}\right)$ and the simplest computation of RWB $\left(C_{\mathcal{I}}^{\Phi}\right)$ can detect QPC and discriminate it from linear and QPU. On the contrary, WB $\left(C_{\mathcal{I}}\right)$ fails to discriminate between QPC and QPU since high values are maintained in both cases. However, none of the normalized domains is able to quantify the strength of QPC, since all are insensitive to changes in $\varepsilon$, while the parameter $E_{\mathcal{I}}$ can be used to quantify the strength of QPC, once that it has been detected. Lower values of $E_{\mathrm{L}, \mathrm{R}}$ (Fig. 4(c)) are due to lower frequency resolution which affects the phase estimation at lower frequencies. Although $E_{\mathcal{I}}$ underestimates QPC strength, it is able to track its changes. 

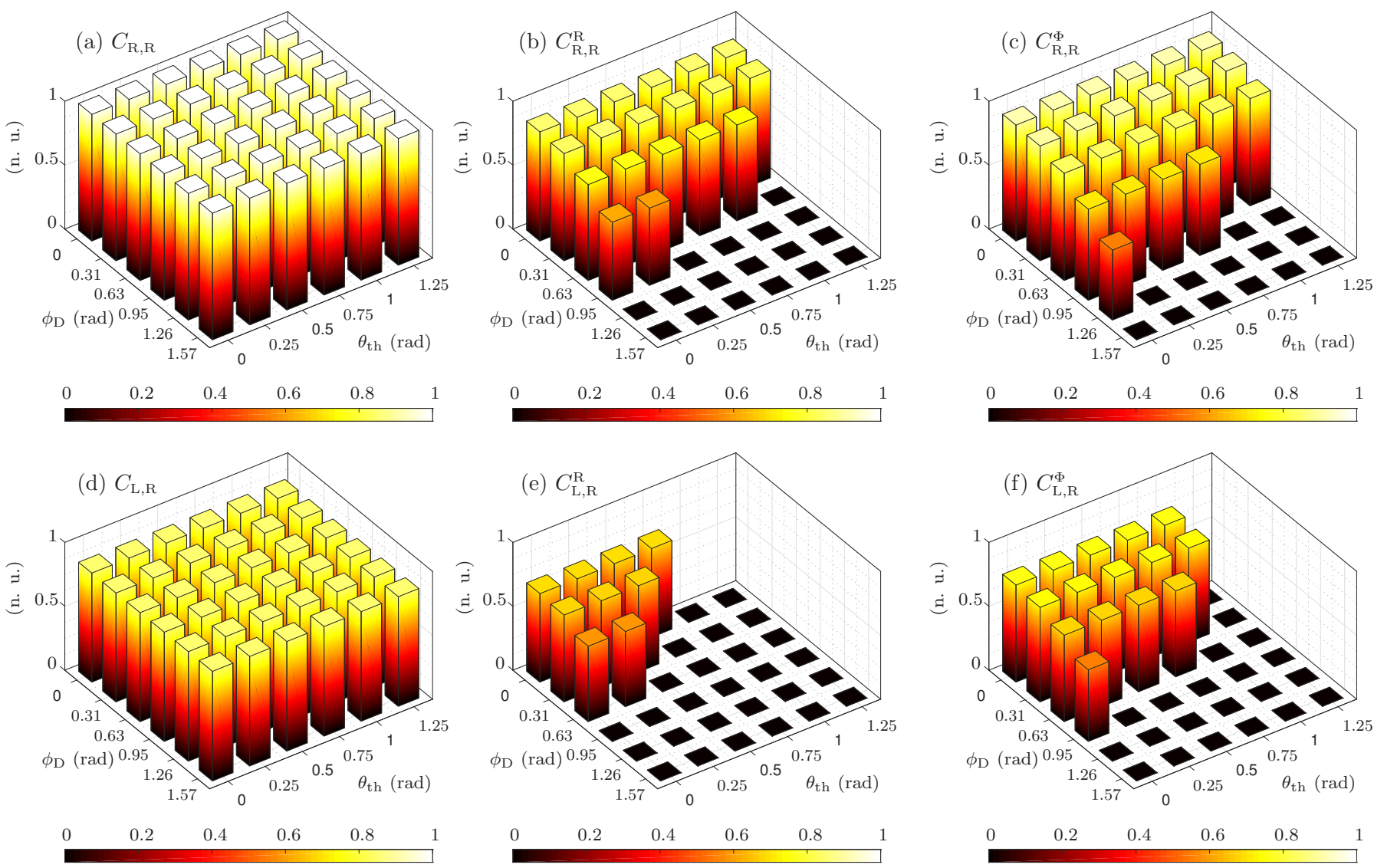

Fig. 3. Influence of delay $\phi_{\mathrm{D}}$ and $\theta_{\mathrm{th}}$ of surrogate analysis in QPC detection for (a) WB, (b) BRWB, (c) RWB in $\Omega_{\mathrm{R}, \mathrm{R}}$, (d) WB, (e) BRWB, (f) RWB in $\Omega_{\mathrm{L}, \mathrm{R}}$.
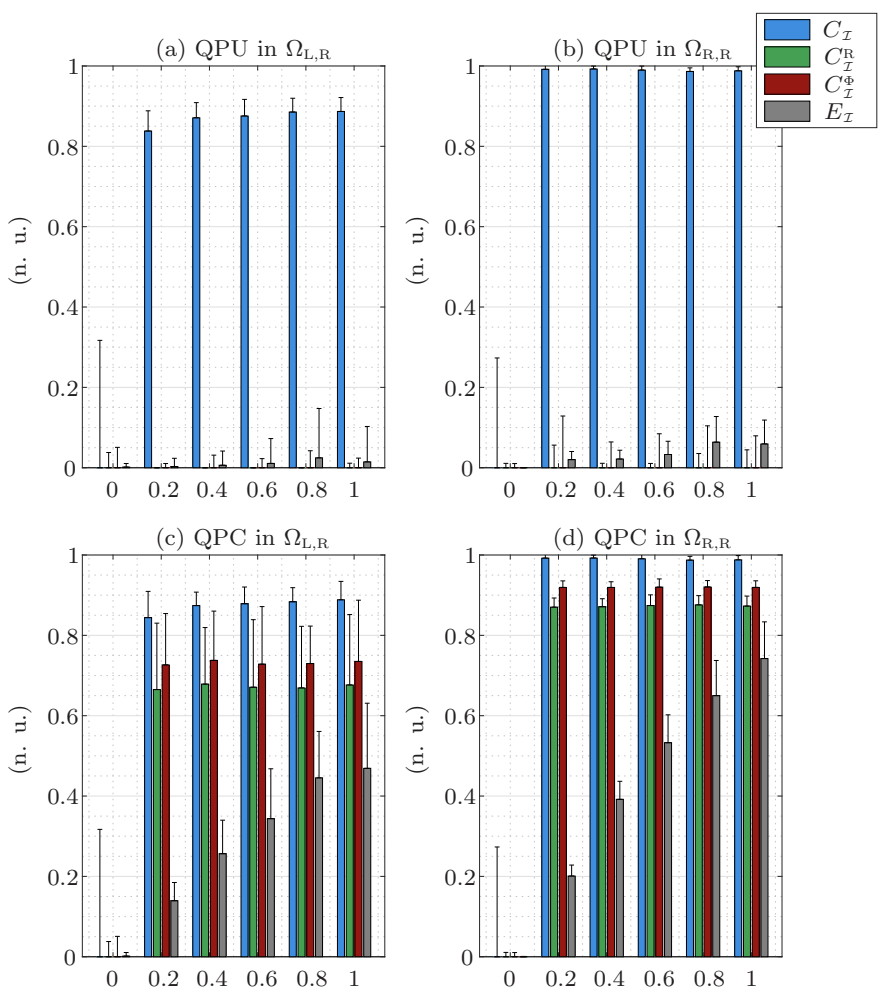

Fig. 4. (a) Estimated coupling for QPU conditions at (a) $\Omega_{\mathrm{L}, \mathrm{R}}$, (b) $\Omega_{\mathrm{R}, \mathrm{R}}$, estimated coupling for QPC conditions at (c) $\Omega_{\mathrm{L}, \mathrm{R}}$, (d) $\Omega_{\mathrm{R}, \mathrm{R}}$.
3) Time-varying QPC tracking: Fig. 5(a) shows a representative example of a simulated HRV signal, whose various frequency components and their evolution in time can be seen in Fig. 5(b). RWB detector $\left(C^{\Phi}(t)\right)$ gets low median values for non-QPC waves (Linear, QPU) and could be used for QPC tracking in weak couplings and even in the presence of a certain delay $\left(\mathrm{QPC}_{\mathrm{D}}\right)$ in the interacting components of HRV (Fig. 5(c)). As it can be appreciated in Fig. 5(d), the median values of estimated bi-frequencies $\left(\hat{f}_{1}, \hat{f}_{2}\right)$, which correspond to the maximum peak in $b_{\Phi}^{2}\left(\hat{f}_{1}, \hat{f}_{2}, t\right)$, are well detected and close to the simulated ones $\left(f_{\mathrm{L}}=0.1 \mathrm{~Hz}, f_{\mathrm{R}}=0.25 \mathrm{~Hz}\right)$. Regarding QPC quantification, median $E(t)$ (Fig. 5(e)) measurements follow the changes of cardiorespiratory coupling across the time. Note that some of the cross-coupling products between sympathetic $\left(f_{1}=0.1 \mathrm{~Hz}\right)$ and parasympathetic branches $\left(f_{2}=0.25 \mathrm{~Hz}\right)$ were located at frequencies close to the one of linear components $\left(f_{2}-f_{1}=0.15 \mathrm{~Hz}\right)$, which may explain the underestimation of coupling strength in the intervals 145$170 \mathrm{~s}$ and 235-280 s (Fig. 5(e)). Those intervals implicate components of low frequencies in $\mathrm{HRV}\left(f_{1}=0.1 \mathrm{~Hz}\right)$ where the frequency resolution of the wavelet is lower.

\section{B. Statistical Analysis of Cardiorespiratory Coupling during Tilt Table Test}

Fig. 6 illustrates the instantaneous QPC quantification for one subject between LF and HF components of HRV and the respiration signal. Row signals are also plotted on the 


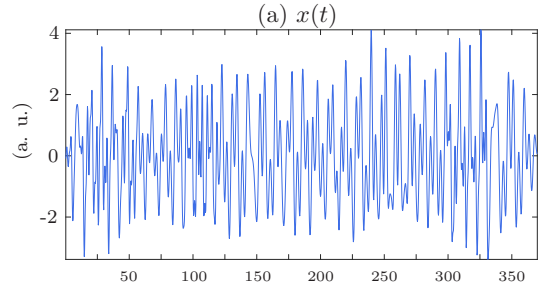

(b) $\left|W_{x}(f, t)\right|$

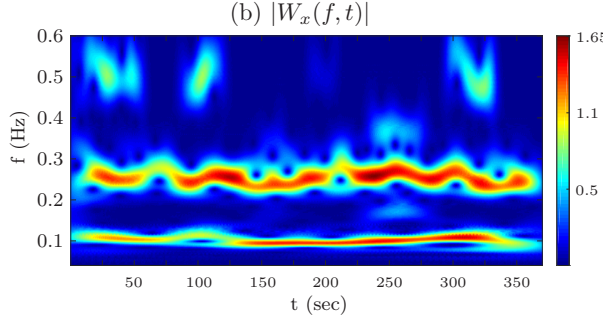

(c) QPC detection
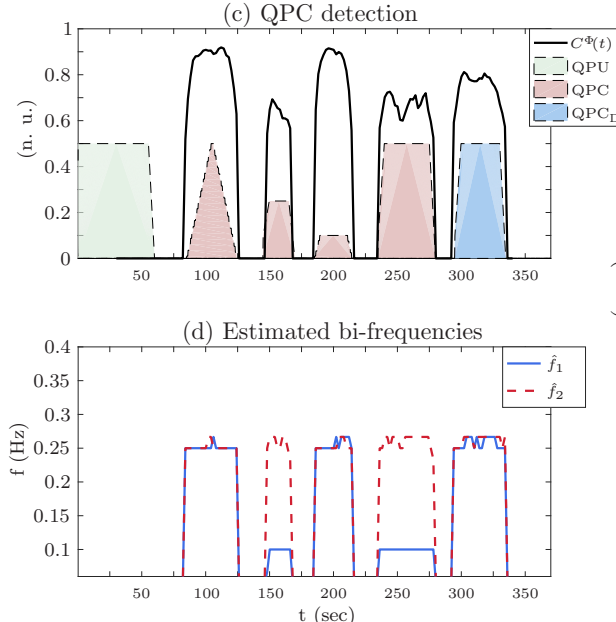

(e) QPC quantification

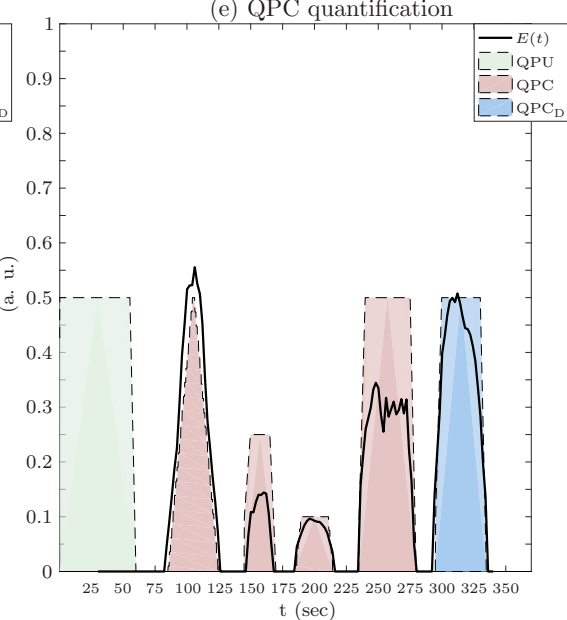

Fig. 5. (a) Synthesized HRV signal $x(t)$, (b) CWT of HRV , (c) Instantaneous cross-RWB QPC detection $\left(C^{\Phi}(t)\right)$, (d) Estimated bi-frequencies $\left(\hat{f}_{1}, \hat{f}_{2}\right)$, which correspond to $C^{\Phi}(t)$, (e) Normalized Wavelet Biamplitude $(E(t))$ for $x(t)$.

(a) $E_{\mathrm{R}, \mathrm{R}}(t)$

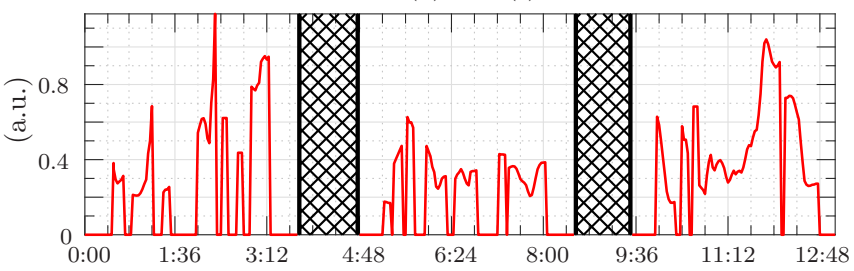

(b) $E_{\mathrm{L}, \mathrm{R}}(t)$

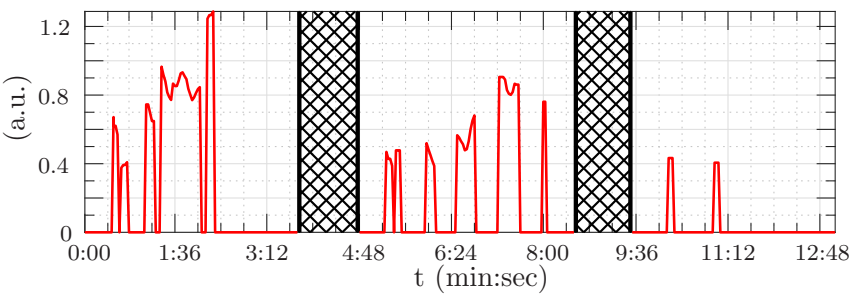

(c) $y(t)$

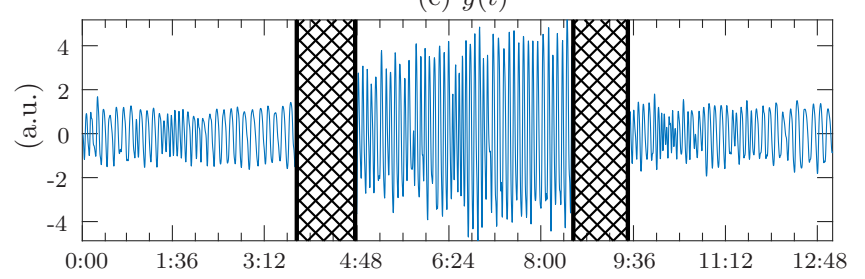

(d) $x(t)$

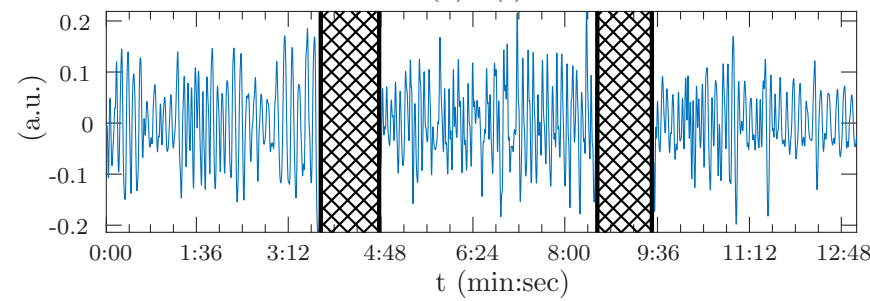

Fig. 6. For a subject of tilt test database (a) Normalized QPC between HF of $x(t)$ and $y(t)$, (b) Normalized QPC between LF of $x(t)$ and $y(t)$, (c) Respiration signal $y(t)$, (d) HRV signal $x(t)$. The black-patterned regions correspond to the beginning and end of $T_{h}$ stage, which were excluded.

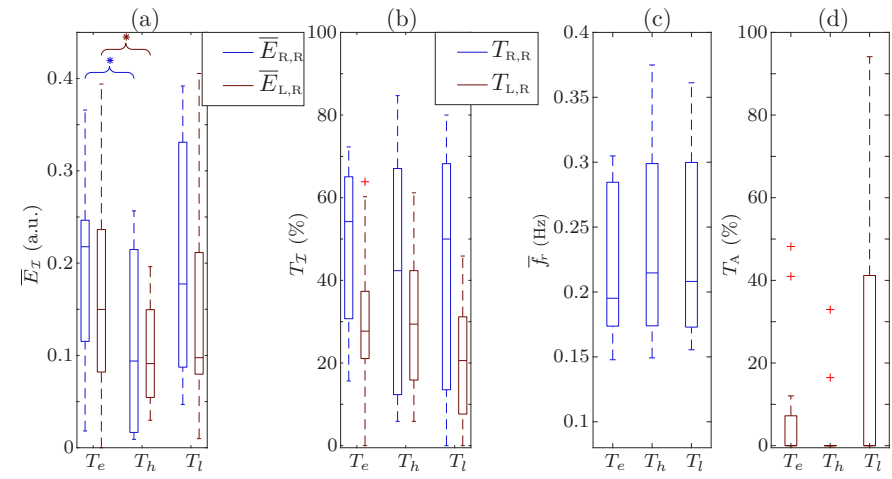

Fig. 7. Boxplots of the feature set together with the aliasing time. (a) Mean NWB for all subregions, (b) Time percentage wherein QPC was present, (c) Mean respiratory rate, (d) Time percentage of aliasing. The statistical differences are marked with an asterisk $(p<0.05)$.

right. Fig. 7 represents the results obtained for the whole database including boxplots for all the subjects with the mean
NWB values for each stage (Fig. 7(a)), the corresponding time percentages wherein QPC was present (Fig. 7(b)), the mean respiratory rate (Fig. 7(c)) for each stage and the time percentages $T_{\mathrm{A}}$ (Fig. $\left.7(\mathrm{~d})\right)$, when aliasing $\left(f_{1}+f_{2}>f_{\mathrm{HR}} / 2\right)$ was present. Statistical differences are marked with an asterisk. The null hypothesis was rejected $(p<0.05)$ for the mean NWB values $\left(\bar{E}_{\mathrm{R}, \mathrm{R}}, \bar{E}_{\mathrm{L}, \mathrm{R}}\right)$ in tilt stage $\left(T_{h}\right.$ vs $\left.T_{e}\right)$, while for the rest of features there were not statistical differences.

\section{DISCUSSION}

Assessment of QPC between respiration and HRV could provide valuable information regarding ANS regulation, apart from RSA which is often used as an index of vagal control of the heart [54], although partially incomplete. Our hypothesis is that HRV is formed as the output of a quadratic-linear system with input respiratory oscillations, among others.

In our work, reliability of QPC detection was evaluated using a HOS-surrogate analysis. The feasibility of different methods in QPC detection was tested, even in the presence 
of constant delay between the interacting oscillations (Fig. 3). WB method seems to ignore phase alternations and thus cannot be used to estimate properly QPC. In BRWB the maximum phase shift for the nonlinear coupling $x_{\mathrm{L}}\left(t-t_{\mathrm{L}}\right) x_{\mathrm{R}}\left(t-t_{\mathrm{R}}\right)$ is $\phi_{\mathrm{D}} \approx 0.63 \mathrm{rad}$, which corresponds to delay parameters $t_{\mathrm{L}} \approx 0.97 \mathrm{~s}, t_{\mathrm{R}} \approx 0.39 \mathrm{~s}$, while for $\mathrm{RWB}$ are $t_{\mathrm{L}} \approx 1.53$ $\mathrm{s}$ and $t_{\mathrm{R}} \approx 0.61 \mathrm{~s}\left(\phi_{\mathrm{D}} \approx 0.95 \mathrm{rad}\right)$. Regarding nonlinear interactions due to the vagal activity $x_{\mathrm{R}}^{2}\left(t-t_{\mathrm{R}}\right)$, in BRWB the maximum delay is approximately $t_{\mathrm{R}} \approx 0.61 \mathrm{~s}\left(\phi_{\mathrm{D}} \approx 0.95\right.$ $\mathrm{rad})$, while a better performance, $t_{\mathrm{R}} \approx 0.77 \mathrm{~s}\left(\phi_{\mathrm{D}} \approx 1.26\right.$ $\mathrm{rad})$, is achieved with RWB. These values are in accordance with physiological responses reported in the literature. For example, the response time of a single efferent vagal impulse on the sinus node is very short and affects only 1 or 2 heartbeats after its onset [46]. Considering a vagal arterial baroreflex mechanism, the latencies between systolic pressure fluctuations and R-R intervals at low frequencies are up to 2.0 $\mathrm{s}$, while average latencies between systolic pressures and R-R intervals at respiratory frequencies are much less $(<0.5 \mathrm{~s})$ [55]. Delay values in those ranges have been used in the literature for modeling a transfer function between instantaneous vagal firing rate and the heart rate [56].

The simplest computation of RWB can be used to detect QPC even for weak couplings and constant delays between interacting oscillations, while the proposed NWB is able to track changes in coupling strength (Fig. 4). In this study the complex Morlet wavelet was used, but either different mother wavelets could be used optimizing their parameters to achieve better frequency resolution, or improved estimation of phase and frequency extraction can be obtained through the synchrosqueezed wavelet transform [30]. Moreover, in a time-varying QPC scenario (Fig. 5), RWB and NWB showed a robust performance. The simulation was carried out in a control manner wherein the same power of linear oscillations (sympathetic and parasympathetic branches) maintain across time and continuous changes on cardiorespiratory coupling strength and delays between components were induced. The usage of RWB was essential for a correct identification of those bi-frequencies with significant QPC level, discriminating them from QPU and linear, while NWB for quantification of time-varying, constant or in presence of delay QPC strength.

The proposed methods were used to study cardiorespiratory nonlinear interactions during ANS changes induced by a tilt table test. NWB (Fig. 7(a)) was significantly reduced in headup tilt position $T_{h}$ with respect to early supine $T_{e}$ for both regions $\Omega_{\mathrm{R}, \mathrm{R}}$ and $\Omega_{\mathrm{L}, \mathrm{R}}(p<0.05)$. Gil et al. [57] reported for the same database that during tilt, the power content in the HF band of HRV did not present any significant change, while the power content in the LF band was significantly higher. The reduction of nonlinear coupling strength between the dominant respiratory and RSA component of HRV $\left(\bar{E}_{\mathrm{R}, \mathrm{R}}\right)$ could be due to a possible vagal withdrawal as previous methods have shown [58], while changes at $\bar{E}_{\mathrm{L}, \mathrm{R}}$ may occur by reciprocal modulation of sympathetic and parasympathetic-related rhythms, which have already been seen on the discharge of central neurons and in peripheral neural outflows [59]. Furthermore, the feedback from pulmonary stretch receptors and direct respiratory-related changes in venous return during head-tilt position could evoke respiratory related fluctuations in HRV, probably through nonlinear processes [60]. Our findings based on NWB provide complementary information to the work Orini et al. [61], who reported for the same database, the absence of linear cardiorespiratory coupling reduction during $T_{h}$. Among the other studied parameters, the mean respiratory rate $\bar{f}_{r}$ (Fig. 7(c)) did not change significantly during the protocol phases while in Fig. 7(b), it can be seen that QPC in $\Omega_{R, R}$ was present for a larger time percentage than $\Omega_{L, R}$. However, the changes at their durations were not found significantly different between stages.

In this study two main contributions, not yet addressed in previous studies on bispectral analysis, are included. First, RWB is introduced simplifying the computation load associated to BRWB, when implementing the phase randomization procedure. In addition, RWB results are more robust than BRWB in the presence of delays between the interacting components under analysis. Finally, a new metric named NWB is introduced, which after detecting the presence of QPC, quantifies its strength. NWB showed to be the most significant feature for distinguishing stages with different ANS regulation during a tilt table test protocol.

One limitation of this study is the exclusion of those peaks where $f_{1}+f_{2}$ was higher than half of mean HR. Thus, the time with no QPC can be attributed to real lack of QPC or to the fact that the coupling is not measurable due to aliasing. In our study the median value of the time percentage where aliasing was present $\left(T_{\mathrm{A}}\right)$ is zero for all the stages (Fig. 7(d)). In some subjects in late supine $\left(T_{l}\right)$, the heart rhythm slows but the respiratory rate is maintained high and thus the time percentage of aliasing is much higher. The percentages of the subjects without any presence of aliasing at each of the protocol stages $T_{e}, T_{h}, T_{l}$ were $65 \%, 82.5 \%$, $59 \%$, respectively. Generally, the methods presented in this paper are suited for assessment of quadratic nonlinearities and could be easily extended to other higher order statistics (cubic) but cannot be used to detect other general forms of nonlinear couplings [26], [30], [58].

\section{CONCLUSION}

A new proposal, namely Real Wavelet Biphase (RWB), in combination with a surrogate data analysis, has been presented for identification of quadratic nonlinear interactions between respiration and HRV signals. The coupling strength of these interactions is quantified based on the Normalized Wavelet Biamplitude (NWB). A variety of simulations were performed to prove RWB reliability and robustness to discriminate Quadratic Phase Coupling, even in the presence of constant delay between interacting oscillations, from Quadratic Phase Uncoupling and Linear relationships. To study cardiorespiratory interactions during a tilt table test, QPC was detected first by the proposed RWB and its strength was quantified based on NWB. Results from the database showed that a tilt test maneuver may lead to changes of quadratic nonlinear interactions of Autonomic Nervous System, since a significant reduction $(p<0.05)$ of NWB was found during the tilted phase compared to the previous phase in supine position. 


\section{REFERENCES}

[1] F. Yasuma and J. Hayano, "Respiratory sinus arrhythmia: why does the heartbeat synchronize with respiratory rhythm?," Chest J., vol. 125, no. 2, pp. 683-690, 2004.

[2] M. B. Daly, "Interactions between respiration and circulation," Compr. Physiol., 1986.

[3] F. Weise and F. Heydenreich, "Effects of modified respiratory rhythm on heart rate variability during active orthostatic load," Biomed. Biochim. Acta., vol. 48, no. 8, pp. 549-556, 1988 .

[4] F. Lombardi, "Clinical implications of present physiological understanding of hrv components," Card. Electrophysiol. Rev., vol. 6, no. 3, pp. 245-249, 2002.

[5] J. P. Saul et al., "Transfer function analysis of the circulation: unique insights into cardiovascular regulation," Am. J. Physiol. Heart Circ. Physiol., vol. 261, no. 4, pp. H1231-H1245, 1991.

[6] R. Bates et al., "Autonomic function assessment using analysis of heart rate variability," Control. Eng. Pract., vol. 5, no. 12, pp. 1731-1737, 1997.

[7] P. C. Ivanov et al., "Multifractality in human heartbeat dynamics," Nature, vol. 399, no. 6735, pp. 461-465, 1999.

[8] F. Peña, "Contribution of pacemaker neurons to respiratory rhythms generation in vitro," Integration Respiratory Control. Springer, pp. 114 118, 2008.

[9] C. A. Del Negro et al., "Models of respiratory rhythm generation in the pre-bötzinger complex. iii. experimental tests of model predictions," $J$. Neurophysiol., vol. 86, no. 1, pp. 59-74, 2001.

[10] P. M. Lehrer and R. Gevirtz, "Heart rate variability biofeedback: how and why does it work?," Front. Psychol., vol. 5, p. 756, 2014.

[11] S. C. Malpas, "The rhythmicity of sympathetic nerve activity," Prog. Neurobiol., vol. 56, no. 1, pp. 65-96, 1998.

[12] D. J. Moraes et al., "Specific respiratory neuron types have increased excitability that drive presympathetic neurones in neurogenic hypertension," Hypertension, vol. 63, no. 6, pp. 1309-1318, 2014.

[13] A. J. Verberne and N. C. Owens, "Cortical modulation of the cardiovascular system," Prog. Neurobiol., vol. 54, no. 2, pp. 149-168, 1998.

[14] C. Schäfer et al., "Synchronization in the human cardiorespiratory system," Phys. Rev. E., vol. 60, no. 1, p. 857, 1999.

[15] B. Musizza et al.,"Interactions between cardiac, respiratory and eeg- $\delta$ oscillations in rats during anaesthesia," J. Physiol., vol. 580, no. 1, pp. 315-326, 2007.

[16] A. Stefanovska et al., "The cardiovascular system as coupled oscillators?," Physiol. Meas., vol. 22, no. 3, p. 535, 2001.

[17] J. Jamšek et al., "Nonlinear cardio-respiratory interactions revealed by time-phase bispectral analysis," Phys. Med. Biol., vol. 49, no. 18, p. 4407, 2004

[18] D. Hoyer et al., "Determinism in bivariate cardiorespiratory phase-space sets," IEEE Eng. Med. Biol. Mag., vol. 17, no. 6, pp. 26-31, 1998.

[19] F. Censi et al., "Nonlinear coupling among heart rate, blood pressure, and respiration in patients susceptible to neuromediated syncope," Ann. Biomed. Eng., vol. 31, no. 9, pp. 1097-1105, 2003.

[20] F. Censi et al., "Spontaneous and forced non-linear oscillations in heart period: role of the sino-atrial node," Med. Eng. Phys., vol. 24, no. 1, pp. 61-69, 2002.

[21] L. Faes and G. Nollo, "Bivariate nonlinear prediction to quantify the strength of complex dynamical interactions in short-term cardiovascular variability," Med. Biol. Eng. Comput., vol. 44, no. 5, pp. 383-392, 2006.

[22] D. Widjaja et al., "Cardiorespiratory information dynamics during mental arithmetic and sustained attention," PloS one, vol. 10, no. 6, p. e0129112, 2015.

[23] M. Baumert et al., "Joint symbolic dynamics for the assessment of cardiovascular and cardiorespiratory interactions," Phil. Trans. R. Soc. A: Mathematical, Physical and Eng. Sciences, vol. 373, no. 2034, p.20140097, 2015.

[24] H. K. Lackner et al., "Phase synchronization of hemodynamic variables and respiration during mental challenge," Int. J. Psychophysiol., vol. 79, no. 3, pp. 401-409, 2011

[25] F. Censi et al., "Coupling patterns between spontaneous rhythms and respiration in cardiovascular variability signals," Comput. Methods Programs Biomed., vol. 68, no. 1, pp. 37-47, 2002.

[26] L. Faes et al., "Information domain approach to the investigation of cardio-vascular, cardio-pulmonary, and vasculo-pulmonary causal couplings," Eng. Approaches Study Cardiovascular Physiology: Modeling, Estimation, and Signal Process., p. 99, 2011.

[27] L. Faes et al., "Information decomposition in bivariate systems: theory and application to cardiorespiratory dynamics," Entropy, vol. 17, no. 1, pp. 277-303, 2015.
[28] M. G. Rosenblum et al., "Identification of coupling direction: application to cardiorespiratory interaction," Phys. Rev. E., vol. 65, no. 4, p. 041909 , 2002.

[29] M. Paluš and A. Stefanovska, "Direction of coupling from phases of interacting oscillators: An information-theoretic approach," Phys. Rev. E., vol. 67, no. 5, p. 055201, 2003.

[30] D. Iatsenko et al., "Evolution of cardiorespiratory interactions with age," Phil. Trans. R. Soc. A, vol. 371, no. 1997, p. 20110622, 2013.

[31] K. C. Chua et al., "Application of higher order statistics/spectra in biomedical signals-a review," Med. Eng. Phys., vol. 32, no. 7, pp. 679689, 2010.

[32] I. Pinhas et al., "Bicoherence analysis of new cardiovascular spectral components observed in heart-transplant patients: statistical approach for bicoherence thresholding," IEEE Trans. Biomed. Eng., vol. 51, no. 10 pp. 1774-1783, 2004.

[33] C. K. Chua et al., "Cardiac health diagnosis using higher order spectra and support vector machine," Open Med. Inform. J., vol. 3, p. 1, 2009.

[34] H. Witte et al., "Technique for the quantification of transient quadratic phase couplings between heart rate components-verfahren zur quantifizierung transienter quadratischer phasenkopplungen zwischen herzfrequenzkomponenten," Biomed. Tech./Biomed. Eng., vol. 46, no. 3, pp. 42-49, 2001.

[35] B. P. Van Milligen et al., "Wavelet bicoherence: a new turbulence analysis tool," Phys. Plasmas, vol. 2, no. 8, pp. 3017-3032, 1995.

[36] I. I. Jouny and R. L. Moses, "The bispectrum of complex signals: definitions and properties," IEEE Trans. Sig. Process., vol. 40, no. 11, pp. 2833-2836, 1992.

[37] S. Elgar and G. Sebert, "Statistics of bicoherence and biphase," $J$ Geophys. Res., vol. 94, no. C8, pp. 10 993-10 998, 1989.

[38] Y. Li et al., "Biphase randomization wavelet bicoherence for mechanical fault diagnosis," Measurement, vol. 49, pp. 407-420, 2014.

[39] H. Farid, "Detecting digital forgeries using bispectral analysis," 1999.

[40] C. Torrence and G. P. Compo, "A practical guide to wavelet analysis," B. Am. Meteorol. Soc., vol. 79, no. 1, pp. 61-78, 1998.

[41] W. Collis et al., "Higher-order spectra: the bispectrum and trispectrum," Mech Syst Signal Process, vol. 12, no. 3, pp. 375-394, 1998.

[42] T. Kim et al., "A novel qpe detector for the health monitoring of rotating machines," IMTC. IEEE, pp. 1-6, 2007.

[43] J. Fackrell et al., "Practical issues concerning the use of the bicoherence for the detection of quadratic phase coupling," Proc. IEEE Workshop HOS, Begur, Girona, Spain, pp. 310-314, 1995.

[44] J. Theiler et al., "Testing for nonlinearity in time series: the method of surrogate data," Physica D, vol. 58, no. 1-4, pp. 77-94, 1992.

[45] C. G. Scully et al., "Detecting interactions between the renal autoregulation mechanisms in time and space," IEEE Trans. on Biomed. Eng, vol. 64 , no. 3, pp. 690-698, 2017.

[46] R. Hainsworth, "Physiology of the cardiac autonomic system," in Clinical guide to cardiac autonomic tests, M. Malik (Eds), Springer, pp. 3-28, 1998.

[47] P. Laguna et al., "Power spectral density of unevenly sampled data by least-square analysis: performance and application to heart rate signals," IEEE Trans. Biomed. Eng., vol. 45, no. 6, pp. 698-715, 1998.

[48] R. Bailón et al., "Analysis of heart rate variability using time-varying frequency bands based on respiratory frequency," in 29th Annu. Int. Conf. IEEE EMBS, pp. 6674-6677, 2007.

[49] A. Hernando et al., "Inclusion of respiratory frequency information in heart rate variability analysis for stress assessment," IEEE J. Biomed. Health Inform., vol. 20, no. 4, pp. 1016-1025, 2016.

[50] J. Mateo and P. Laguna, "Analysis of heart rate variability in the presence of ectopic beats using the heart timing signal," IEEE Trans. Biomed. Eng., vol. 50, no. 3, pp. 334-343, 2003.

[51] M. Orini et al., "Synthesis of hrv signals characterized by predetermined time-frequency structure by means of time-varying arma models," Biomed. Signal Process. Control, vol. 7, no. 2, pp. 141-150, 2012.

[52] J. Jamšek et al., "Wavelet bispectral analysis for the study of interactions among oscillators whose basic frequencies are significantly time variable," Phys. Rev. E, vol. 76, no. 4, p. 046221, 2007.

[53] J. Jamšek et al., "Detecting couplings between interacting oscillators with time-varying basic frequencies: Instantaneous wavelet bispectrum and information theoretic approach," Phys. Rev. E, vol. 81, no. 3, p. 036207, 2010.

[54] G. G. Berntson et al., "Respiratory sinus arrhythmia: autonomic origins, physiological mechanisms, and psychophysiological implications," $J$. Psychophysiol., vol. 30, no. 2, pp. 183-196, 1993.

[55] D. L. Eckberg, "Physiological basis for human autonomic rhythms," Ann. Med., vol. 32, no. 5, pp. 341-349, 2000. 
[56] J. P. Saul et al., "Transfer function analysis of autonomic regulation. ii. respiratory sinus arrhythmia," Am. J. Physiol. Heart Circ. Physiol., vol. 256, no. 1, pp. H153-H161, 1989.

[57] E. Gil et al., "Photoplethysmography pulse rate variability as a surrogate measurement of heart rate variability during non-stationary conditions," Physiol. Meas., vol. 31, no. 9, pp. 1271-1290, 2010.

[58] A. Porta et al., "Conditional self-entropy and conditional joint transfer entropy in heart period variability during graded postural challenge," PLoS One, vol. 10, no. 7, p. e0132851, 2015.

[59] N. Montano et al., "Presence of vasomotor and respiratory rhythms in the discharge of single medullary neurons involved in the regulation of cardiovascular system," J. Auton. Nerv. Syst., vol. 57, no. 1-2, pp. 116-122, 1996.

[60] D. Mendelowitz, "Advances in parasympathetic control of heart rate and cardiac function," Phys., vol. 14, no. 4, pp. 155-161, 1999.

[61] M. Orini et al., "Characterization of dynamic interactions between cardiovascular signals by time-frequency coherence," IEEE Trans. Biomed. Eng., vol. 59, no. 3, pp. 663-673, 2012. 